\title{
The establishment of a growth-controllable orthotopic bladder cancer model through the down-regulation of c-myc expression
}

\author{
Ho Kyung Seo ${ }^{1,2}$, Seung-Phil Shin², Na-Rae Jung ${ }^{2}$, Whi-An Kwon ${ }^{3}$, Kyung-Chae \\ Jeong ${ }^{4}$ and Sang-Jin Lee ${ }^{2}$ \\ ${ }^{1}$ Center for Prostate Cancer, Hospital, National Cancer Center, Goyang, Gyeonggi-do, Korea \\ ${ }^{2}$ Genitourinary Cancer Branch, Research Institute, National Cancer Center, Goyang, Gyeonggi-do, Korea \\ ${ }^{3}$ Department of Urology, School of Medicine, Institute of Wonkwang Medical Science, Wonkwang University, Wonkwang \\ University Sanbon Hospital, Gunpo, Gyeonggi-do, Korea \\ ${ }^{4}$ Biomolecular Function Research Branch, Research Institute, National Cancer Center, Goyang, Gyeonggi-do, Korea \\ Correspondence to: Sang-Jin Lee, email: leesj@ncc.re.kr \\ Ho Kyung Seo, email: seohk@ncc.re.kr \\ Keywords: bladder cancer, c-myc, orthotopic bladder cancer model, MBT-2
}

Received: April 20, $2016 \quad$ Accepted: June 17, 2016

Published: July 22, 2016

Copyright: Seo et al. This is an open-access article distributed under the terms of the Creative Commons Attribution License 3.0 (CC BY 3.0), which permits unrestricted use, distribution, and reproduction in any medium, provided the original author and source are credited.

\section{ABSTRACT}

To properly evaluate the biological effects of immunotherapy, it is critical to utilize a model of cancer in immune-competent mice. Currently, MBT-2 is the most common murine bladder cancer cell line used in orthotopic bladder cancer models, even though this cell type often has an inappropriate genetic mutation landscape. In these models, after tumors are detected with in vivo imaging, the mouse usually dies within two to three weeks due to post-renal azotemia caused by the rapidly growing mass. This event prohibits the evaluation of tumor behavior upon intravesical drug treatment. We explored whether an shRNA-induced decrease in the expression of the $c-m y c$ oncogene in MBT-2 cells could slow down their in vitro proliferation and in vivo tumor growth. We transduced MBT-2 cells with shRNA lentiviruses that bound c-myc, established MBT2.CMYCshRNA and confirmed the retardation of the growth of tumors implanted in $\mathrm{C} 3 \mathrm{H} / \mathrm{He}$ mice. Accordingly, this study suggests that this novel orthotopic bladder cancer model in immune-competent mice may be more appropriate for the analysis of the effects of the intravesical instillation of immunotherapeutic agents.

\section{INTRODUCTION}

Non-muscle-invasive bladder cancer (NMIBC) accounts for $>70 \%$ of all newly diagnosed cases of bladder cancer. The gold standard for the treatment of patients with NMIBC is transurethral resection. Major obstacles in the treatment of this cancer include the high incidence of tumor recurrence and the progression to muscle-invasive disease. To prevent recurrence and progression, intravesical Bacillus Calmette-Guerin (BCG) immunotherapy or intravesical chemotherapy are used as adjuvant therapies; however, a substantial number of patients do not complete this therapy owing to refractory disease or intolerance [1]. Novel intravesical treatment strategies with improved efficacy and lower toxicity are urgently needed. Immunotherapy holds promise in the battle against late-stage and metastatic bladder cancer, which have a poor prognosis [2]. The discovery of immune checkpoint proteins, such as CTLA-4 (cytotoxic T-lymphocyte-associated protein 4), PD-1 (programmed death-1), TIM-3 (T-cell immunoglobulin and mucindomain containing-3), LAG-3 (Lymphocyte-activation gene 3), and IDO (Indoleamine 2,3-dioxygenase), along with a better understanding of the role that they play in the mechanisms of immune evasion in tumors have facilitated the production of a significant anti-tumor immune response in patients with cancer. For example, the use of monoclonal antibodies to block the inhibitory signals of the programmed death-1 (PD-1)/programmed death-ligand 1 (PD-L1) pathway has demonstrated potential for chemotherapy-resistant patients [2]. A number of other cancer immunotherapies are under investigation, but few immune-competent mouse models for bladder cancer exist. 
To evaluate the effects of new immunotherapeutic modalities, it is essential to use a suitable immune-competent animal model that mimics the behavior of human disease. Several strategies have been developed that focus on the immune response or gene therapy. First, genetically engineered mouse (GEM) models enable the analysis of bladder tumors in immune-competent mice. A number of GEM models of urothelial cell carcinoma (UCC) employ the uroplakin II (UPII) promoter of the mouse UPII gene. By placing the UPII promoter upstream to drive the urotheliumspecific expression of the SV40-T antigen, the GEM develops UCCs that bear a strong resemblance to human UCCs [3]. However, cancer cells in GEM models are less heterogeneous than human bladder cancer cells due to their similar origins, and as a result, GEM cannot be used to test the efficacy of novel immunotherapeutic agents. Alternatively, syngeneic tumors can be established by implanting bladder tumor cells formed by exposure to carcinogens. All studies involving syngeneic models have used MBT-2, MB49 or AY-27 cells [4]. Such syngeneic models exhibit good tumor acceptance rates and human bladder cancer mimicry. However, after the bladder tumors are detected with non-invasive in vivo imaging, such as MRI or bioluminescence imaging, the mouse dies within two to three weeks due to azotemia [5]. This outcome makes it impossible to observe long-term tumor responses after intravesical immunotherapeutic treatments. Therefore, the development of a bladder tumor model in mice with an appropriate growth rate that reflects the slow long-term progression of the disease is required.

The $c-m y c$ gene, which is translated to the c-MYC protein, is amplified and/or up-regulated in many types of cancer cells, and it plays critical roles in their malignant transformation, proliferation, apoptosis and metastasis [6]. It is believed that c-MYC regulates the expression of $15 \%$ of all genes and promotes cell cycle progression [7]. In quiescent cells, c-MYC expression is relatively undetectable. However, mitogenic stimulation rapidly induces the production of $c-m y c$ mRNA and protein and drives cells to enter the G1 phase of the cell cycle. Representative genes enhanced by c-MYC include $c d k 4$ and cyclin D2, which are essential for cell cycle progression [8]. Therefore, c-MYC is a potential target for the control of cancer proliferation. In the present study, we explored whether the moderate siRNA-induced down-regulation of c-myc expression in MBT-2 cells decreases cell proliferation and tumor growth in an orthotopic model of bladder cancer. These results may help facilitate the establishment of an appropriate murine bladder tumor model.

\section{RESULTS}

\section{Establishment of MBT-2 cells expressing luciferase and GFP reporters}

MBT-2 cells are murine bladder cancer cells with epithelial characteristics. Their formation is induced by the administration of FANFT (N-[4-(5-nitro-2-furyl)-2thiazolyl] formamide) to $\mathrm{C} 3 \mathrm{H} / \mathrm{He}$ mice [9]. In the past decade, significant advances have been made in the non-invasive evaluation of tumors within the body. Here, we induced MBT2.Luc cells to express luciferase and GFP to monitor tumor growth and the response to treatment using non-invasive in vivo imaging of the bladder. For this purpose, MBT-2 cells were transduced with lentiviruses that delivered both the luciferase and GFP genes (Figure 1A). MBT-2 cells that expressed GFP were isolated with a flow cytometer to construct MBT2.Luc cells. Luciferase gene expression in MBT-2.Luc cells was confirmed by luminescence. These MBT2.Luc cells highly expressed luciferase (Figure 1B) and were able to form a tumor in the bladder (Figure 1C). The bladder tumors grew quickly, filling more than $90 \%$ of the bladder's volume within 2 weeks (Figure 1D). This result suggested that the use of tumors implanted with MBT-2 and MBT2. Luc cells would not be appropriate to monitor the effects of intravesical treatment.

\section{Cell proliferation was inhibited by siRNA binding c-myc}

The transcription factor $c-m y c$ is up-regulated in many tumors [10-12] and plays critical roles in the progression of cancer cells [13]. In a previous study, we demonstrated that $c$-myc expression can be downregulated to inhibit the in vitro cell proliferation and the in vivo tumor growth of bladder cancer cells [14]. Here, we examined whether siRNA against $c-m y c$ could effectively down-regulate $c-m y c$ expression and slow the proliferation of MBT-2 cells. Upon siRNA transfection, $c-m y c$ expression was suppressed, as confirmed by western blot analysis (Figure 2A). As expected, siRNA against $c-m y c$ significantly slowed cell proliferation rates $(P=0.012)$ relative to control siRNA (Figure 2B). Furthermore, we tested whether the reduced cell proliferation would translate to the in vivo tumor growth in the bladder. The tumor growth of MBT-2 cells transfected with siRNA against $c$-myc was slower than that of those treated with control siRNA (Figure 2C), which confirmed that $c$-myc controls the growth rate of MBT-2 tumors in the bladder.

\section{MBT-2 cells stably expressed shRNA against c-myc}

Next, MBT-2 cells were manipulated to reduce proliferation and consequently decelerate tumor growth. To construct MBT2.Luc cells, we utilized shRNA delivered by lentiviruses. Using a lentiviral vector system developed in a previous report [14], siRNA-inducing shRNA that bound to $c-m y c$ transcript was designed (Figure 2) and cloned into a modified pLoxLL3.7 vector (Figure 3A). Lentiviruses harboring c-MYCshRNA effectively silenced the expression of the $c-m y c$ gene (Figure 3B) and cyclin D2, which is controlled by $c-m y c$ transcription 
factor. These results demonstrated that transcribed shRNA was effectively converted to siRNA to silence c-myc expression. The stable MBT-2 cells expressing c-MYCshRNA were named MBT2.cMYCshRNA cells. To confirm that MBT2.cMYCshRNA showed reduced proliferation, cells were plated and evaluated compared with control shRNA-transfected MBT-2 cells. As shown in Figure 3C, MBT2.cMYCshRNA cells exhibited significantly slower proliferation rates $(P<0.0001)$.

\section{The in vivo growth rate was reduced with the down-regulation of c-myc expression}

To verify tumor formation and the control of the growth rate in the bladder, MBT2.cMYCcshRNA cells were infected with lentiviruses expressing GFP. The resultant cells were named MBT2.cMYCcshRNA.
GFP, and their growth was monitored by fluorescence (Figure 4A). Before exploring the growth of orthotopic xenografts, the tumors of $\mathrm{C} 3 \mathrm{H} / \mathrm{He}$ mice were subcutaneously inoculated with MBT2.cMYCcshRNA. GFP cells and monitored relative to MBT2.ControlshRNA. GFP. As shown in Figure 4A, tumors implanted with MBT2.cMYCcshRNA.GFP grew significantly more slowly than those expressing ControlshRNA $(P<0.0001)$. Similar to the subcutaneous tumors, the syngeneic tumors in the bladder were smaller upon the reduction of $c-m y c$ expression with shRNA (Figure 4B, right) $(P<0.001)$. The region of intensity (ROI) calculations confirmed the lower growth rate of tumors composed of MBT2.cMYCcshRNA. GFP cells (Figure 4B, left). These results were verified by MRI analysis with statistical analysis $(P=0.0429)$, which was employed to visualize the exact size of tumors in the bladder (Figure 4C). The mice expressing

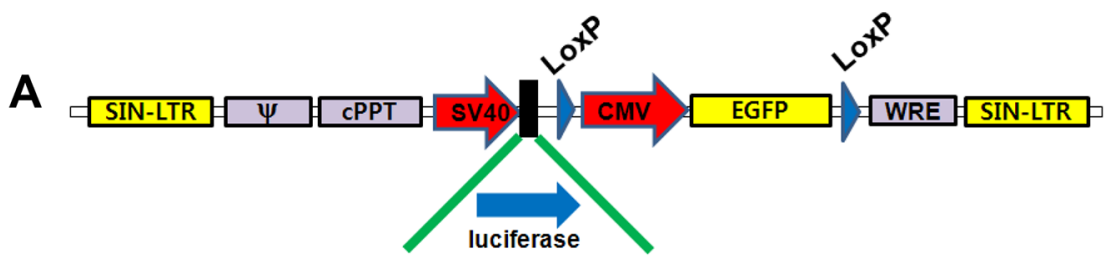

B

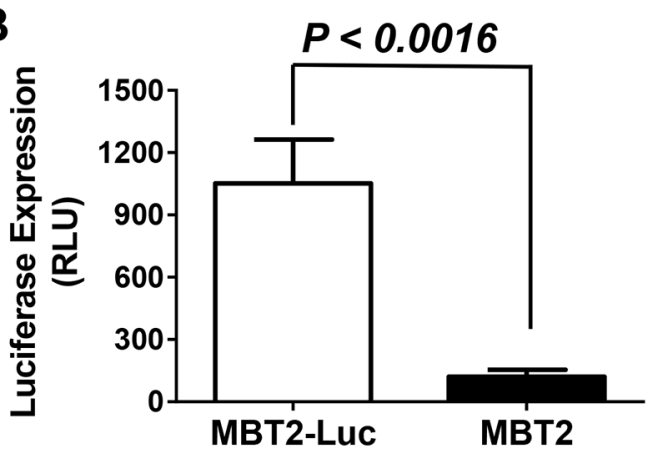

C

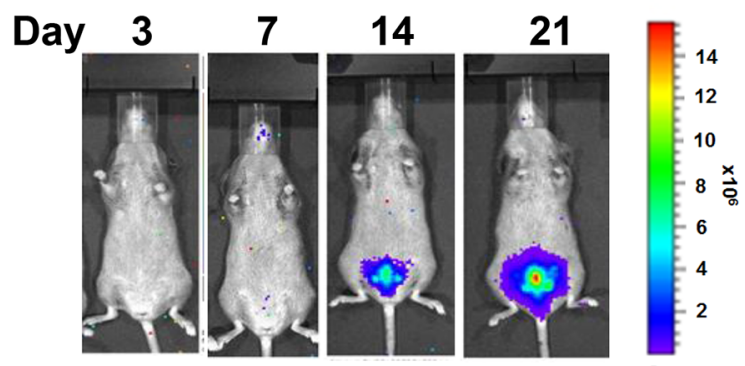

D

Counts

Color Scale

$\operatorname{Max}=1.5445 \mathrm{e}^{+07}$
Man

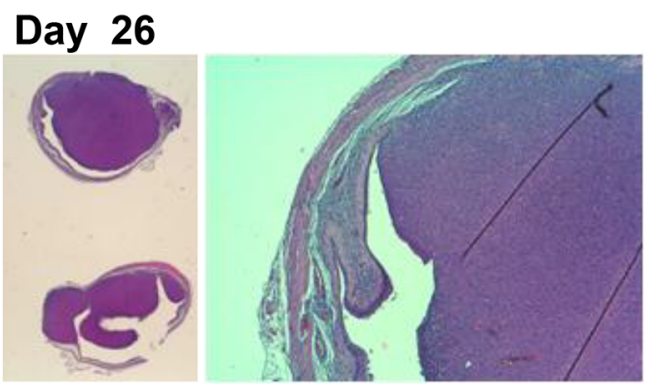

Figure 1: Construction of MBT2.Luc cells expressing the luciferase gene. (A) The lentivirus shuttle vector pLoxLL3.7 was engineered to express a luciferase expression cassette under the SV40 promoter. Then, a four-plasmid-based lentiviral expression system was co-transfected with pMDLg/pRRE, pRSV-Rev and pCMV-G. (B) Approximately $1 \times 10^{5}$ MBT2.Luc and MBT-2 cells were prepared in 12-well plates for $24 \mathrm{~h}$. Cells were harvested for luminescence measurements as described in the Materials and Methods. The data represent the mean \pm SD. (C) To establish the orthotopic bladder cancer model, $50 \mu \mathrm{l}$ of $0.1 \mu \mathrm{g} / \mathrm{mL}$ poly-L-lysine was instilled for 15 minutes, and the bladder was voided. Approximately $2 \times 10^{6}$ cells suspended in PBS were instilled intravesically via the urethra using a 22-gauge arterial puncture needle cannula. Luminescence images were captured at the indicated time points using INVIVO Lumina. (D) For histological analyses, all tumors from five mice were harvested at day 26. Tumor sections (5-10-mm thick) were affixed to slides, de-waxed with ethanol, and stained with hematoxylin and eosin (H\&E). 
either ControlshRNA or cMYCshRNA survived longer when inoculated with MBT2cMYCshRNA.GFP with statistical significance $(P=0.0462)$ (Figure 5). Furthermore, we subcutaneously vaccinated mice with irradiated MBT- 2 cells twice and challenged them with MBT2cMYCshRNA.GFP cells. Upon vaccination, the survival significantly increased with no deaths at 45 days. These results suggest that MBT2.cMYCcshRNA.GFP cells represent a more appropriate murine model for the evaluation of immunotherapeutic treatments.

\section{DISCUSSION}

The efficacy of drug therapies in human cancer patients cannot be reliably assessed with in vitro studies. Animal models are essential to the study of the efficacy of antineoplastic drugs. To effectively evaluate the effect of intravesical therapy in bladder cancer, an animal model must meet several requirements: 1) the tumor is of urothelial origin, with different stages of disease progression; 2) the tumor grows intravesically and can be directly exposed to antineoplastic drugs; and 3) it mimics the clinical course of human urinary bladder cancer. Three types of animal models have been proposed in previous reports: a chemically induced model, an orthotopic transplantable xenograft model, and a genetically engineered model [15]. However, most models are not suitable to evaluate therapy for NMIBC. The induction of primary bladder tumors with carcinogens requires several months, and tissues other than the urothelium can be unintentionally transformed [16]. Therefore, a transplantable orthotopic bladder tumor model is more
A

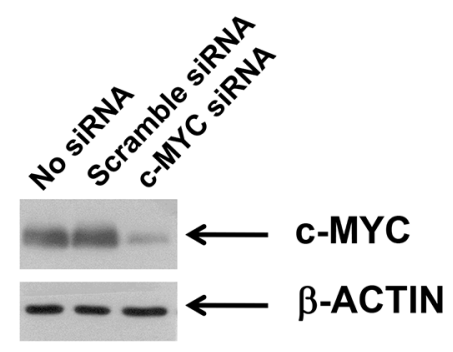

C

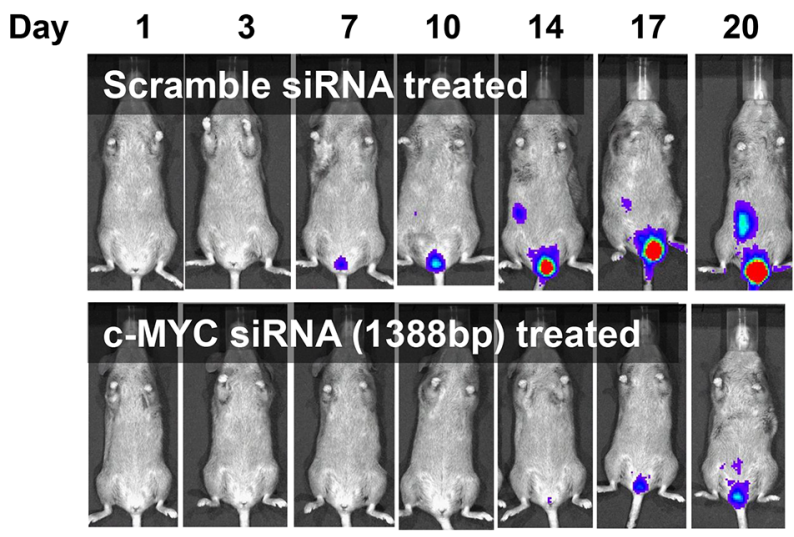

B
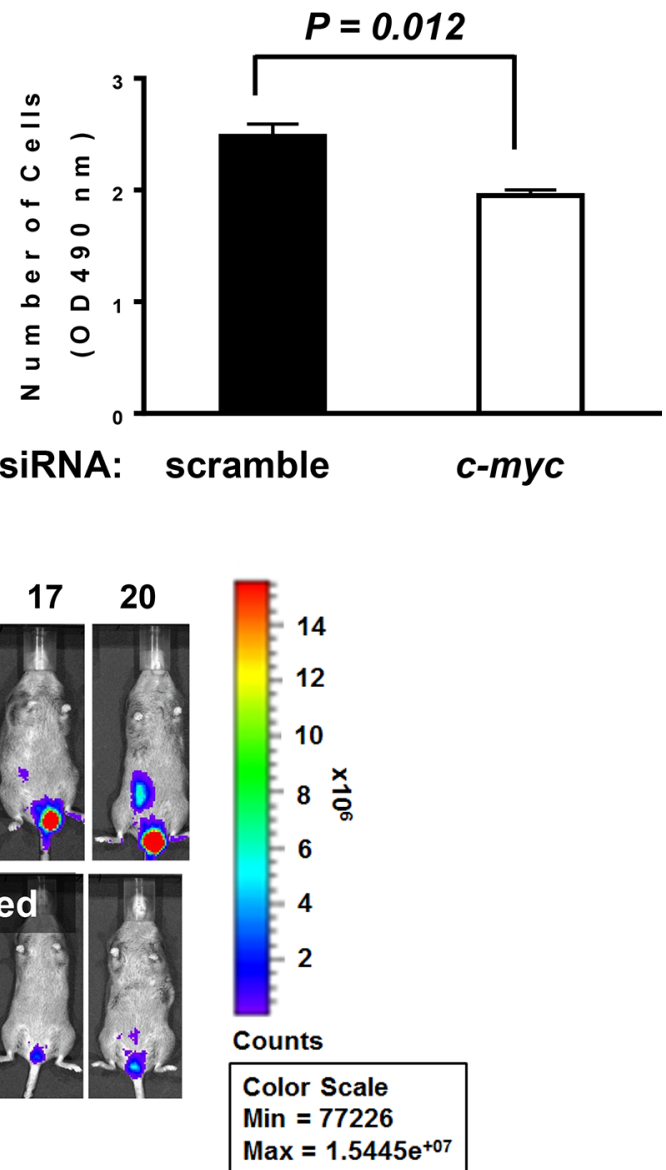

Figure 2: siRNA binding $c$-myc transcripts negatively influenced proliferation. (A). MBT2.Luc cells were seeded at $2.5 \times 10^{5}$ cells/well in 6-well plates to $40-50 \%$ confluence and transiently transfected with $c$-myc siRNA oligonucleotides or control scrambled siRNA. Then, cells were lysed with RIPA buffer. Approximately $30 \mathrm{mg}$ of proteins were resolved on SDS-PAGE and transferred to PVDF for western blot analysis. Membranes were probed with antibodies against c-MYC and the subsequent corresponding secondary antibody was detected using the Enhanced Chemiluminescence (ECL) Plus kit. (B). Each cell line $\left(3 \times 10^{3}\right.$ cells/well) was plated in a 96-well plate for $48 \mathrm{~h}$ and MTT assays were used to count viable cells. The data represent the mean $\pm \mathrm{SD}$. $P$ values were determined by two-tailed paired $t$-tests. $(\mathbf{C})$. MBT2.Luc cells $\left(2 \times 10^{6}\right.$ cells/well $)$ were plated in a 100 -mm dish and transiently transfected with siRNA oligonucleotides binding mouse $c$-myc or scrambled siRNA. Approximately $1 \times 10^{6}$ cells suspended in PBS were instilled in the bladder of a single mouse intravesically using a 22-gauge arterial puncture needle cannula. Luminescence images were taken at the indicated time points using INVIVO Lumina. 
useful for testing new anticancer therapies. Human bladder cancer cell lines can be introduced into the bladders of immunocompromised mice. However, tumors are not reliably acquired with cell lines other than KU7 [17], which are actually HeLa cells [18]. To improve tumor uptake, tumor cells can be directly injected into the bladder wall with or without laparotomy [19]. However, in this model, the tumor's epicenter is located in a muscle layer and grows expansively away from the lumen of the bladder; this growth pattern differs from the pattern of bladder cancer growth in humans, in which tumors originate from the urothelial surface of the bladder and grow intravesically. Moreover, this model is not suitable to evaluate the effect of the intravesical instillation of a novel drug to treat NMIBC because in this model, tumors invade muscle and are covered with normal urothelium, which disturbs the intravesical treatments that must be delivered directly to the tumor. Several studies have utilized orthotopic xenografts of human bladder cancer cells to explore drug efficacy.

Immunotherapy has been an effective treatment for bladder cancer since BCG therapy was introduced
40 years ago [1]. This successful immunotherapy appears to involve various immune cells, such as $\mathrm{CD}^{+}$and $\mathrm{CD}^{+}$lymphocytes, natural killer cells, granulocytes, macrophages, and dendritic cells [20]. Approximately $30 \%$ to $40 \%$ of patients do not respond to this type of therapy [21] due to an inadequate immune response. Recently, antibody-based immunotherapy has emerged as a promising option for bladder cancer. Of the twelve known monoclonal antibodies for cancer, programmed cell death (PD) inhibitors, such as atezolizumab (Roche/Genentech), nivolumab (Bristol-Myers Squibb) and pembrolizumab (Merck), are particularly effective in immunotherapy for bladder cancer. A number of potent immunotherapies, including adoptive cell therapy, vaccines and antibodies, are being investigated worldwide; however, no appropriate immune-competent animal model is currently available. Although a transplantable MBT-2 orthotopic model in $\mathrm{C} 3 \mathrm{H} / \mathrm{HeJ}$ mice has been widely used in BCG-based studies, syngeneic MBT-2 tumors of the bladder grow so rapidly that it is impossible for the immune system to mobilize, which occurs in human bladder cancer patients. However, if the expression of the $c$-myc gene is down-
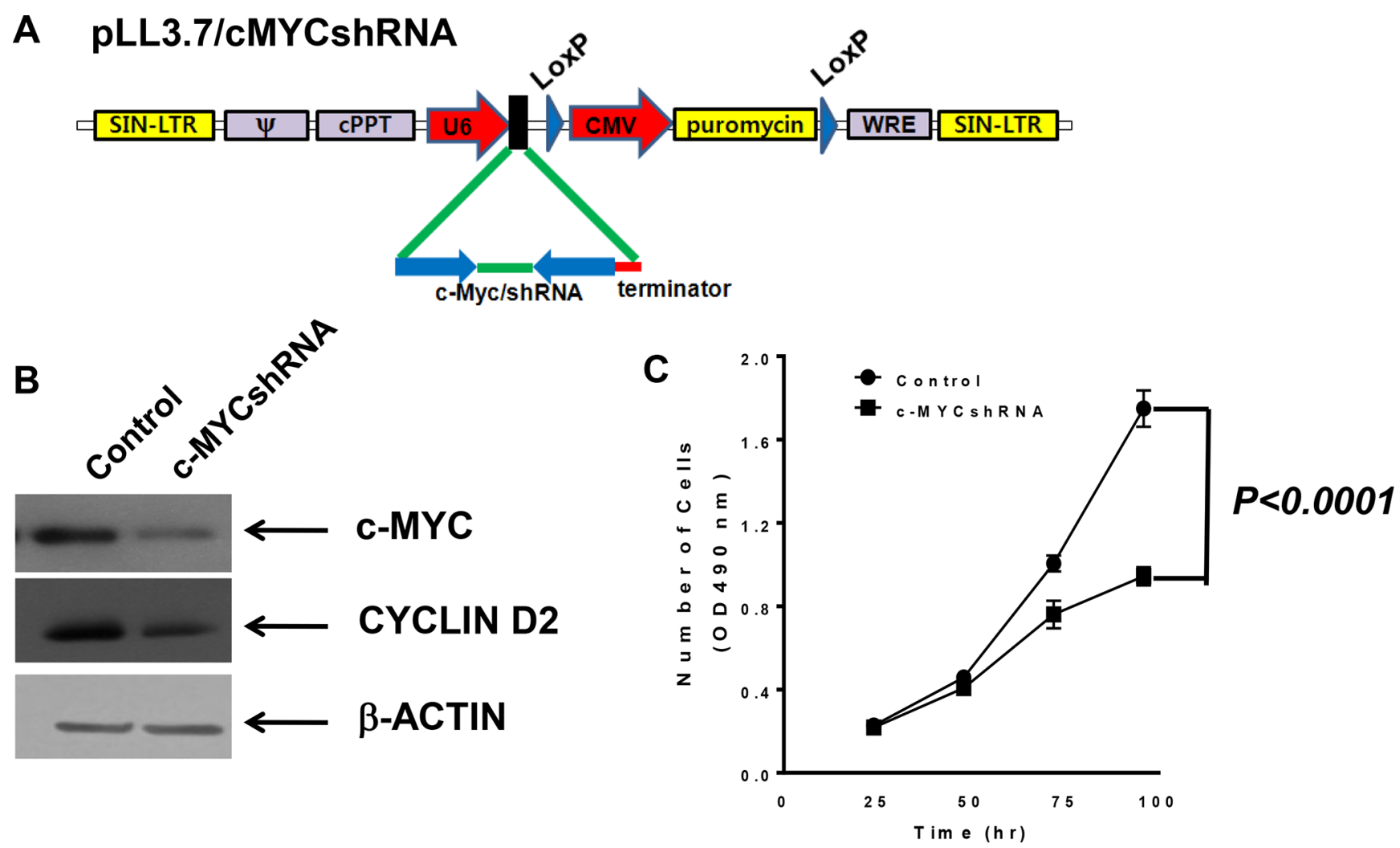

Figure 3: MBT2.cMYCshRNA cells expressing shRNA showed reduced numbers of $\boldsymbol{c}$-myc transcripts. (A) The lentivirus shuttle vector pLoxLL3.7 was engineered to express $c$-myc shRNA under the U6 promoter (pLL3.7/cMYCshRNA). Then, a four-plasmidbased lentiviral expression system was co-transfected with pMDLg/pRRE, pRSV-Rev and pCMV-G. (B) Cell lysates of MBT-2 and MBT2. cMYCshRNA were prepared and resolved on SDS-PAGE for western blotting. Western blotting using c-MYC-reactive or cyclin D2 antibodies showed the reduction of $c-m y c$ mRNA and the down-regulation of the cyclin D2 gene. (C) Approximately $3 \times 10^{3}$ cells of each cell line were plated in a 96-well plate for $48 \mathrm{~h}$, and proliferation was evaluated using MTT assays. $P$ values were determined by two-tailed paired $t$-tests. 

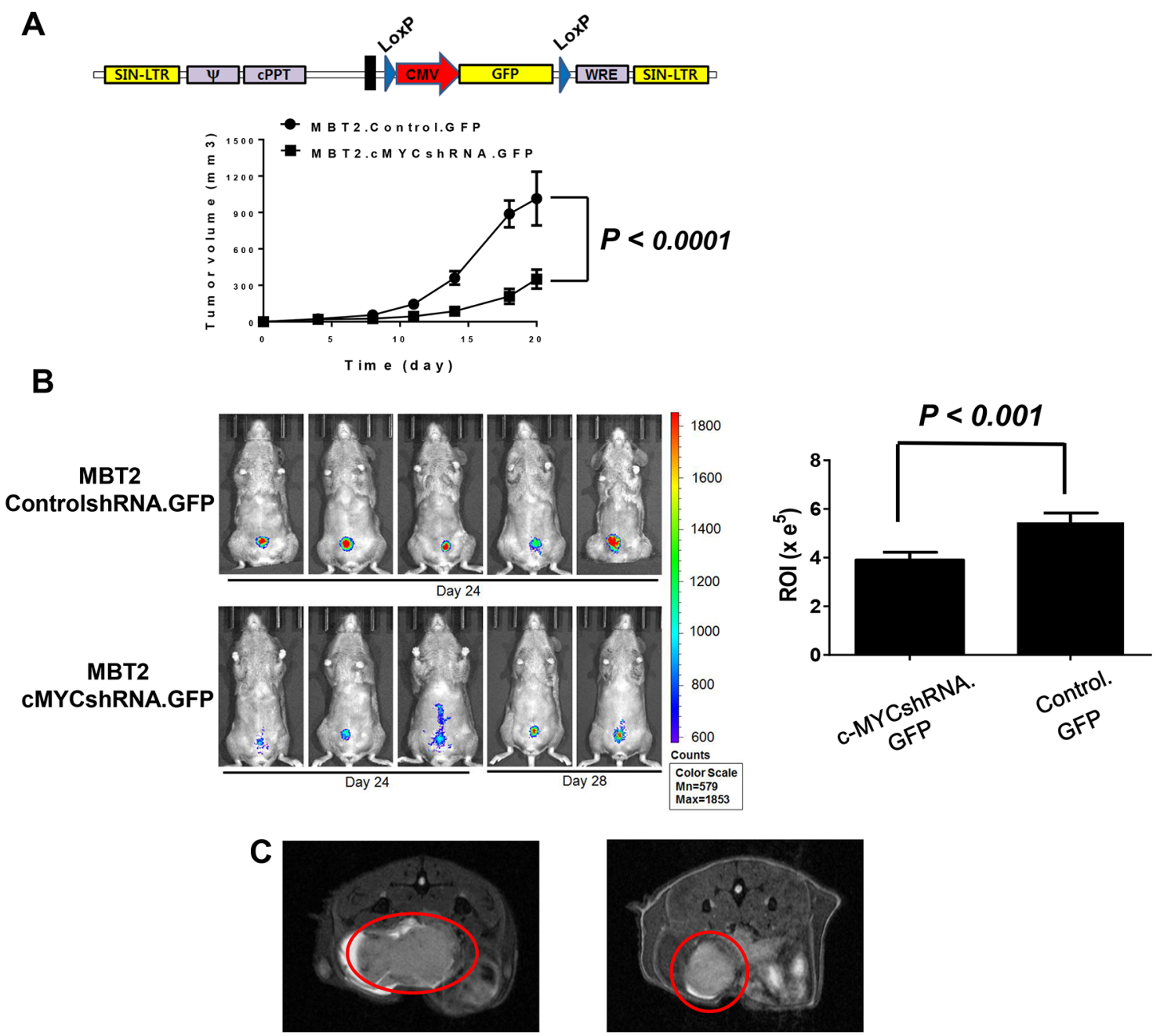

\section{MBT2.Control.GFP MBT2.cMYCshRNA.GFP}

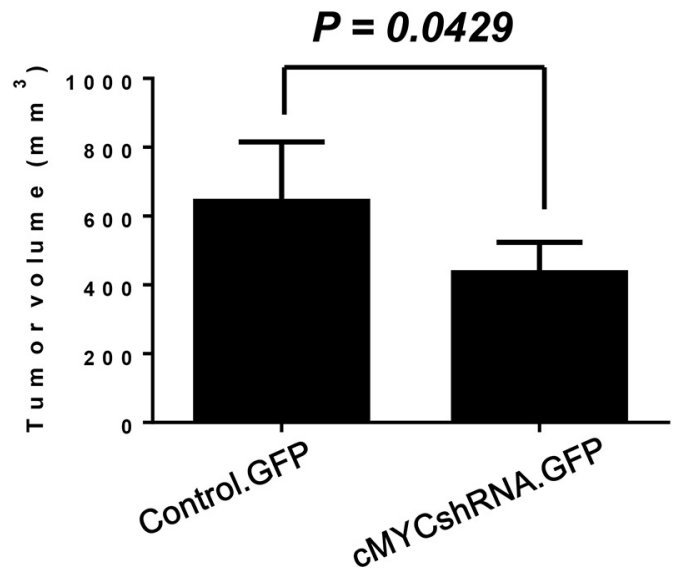

Figure 4: Repressed in vivo tumor growth of MBT2.cMYCshRNA.GFP in the mouse bladder. (A) The lentivirus shuttle vector pLoxLL3.7 was engineered to express the GFP gene under the CMV promoter. Then, cells were infected with lentiviruses expressing GFP and fluorescence-expressing cells were sorted with a flow cytometer. Then, $1 \times 10^{6}$ MBT2.cMYCshRNA.GFP or MBT2. ControlshRNA.GFP cells were subcutaneously implanted in five $\mathrm{C} 3 \mathrm{H} / \mathrm{He}$ mice. The tumor size was measured at the indicated time points. (B) Approximately $1 \times 10^{6}$ cells suspended in PBS were instilled into the bladder intravesically using a 22-gauge arterial puncture needle cannula. Fluorescence images were taken at the indicated time points using INVIVO Lumina, and the ROIs of luminescence surrounding the tumor were calculated. $P$ values were determined by two-tailed paired $t$-tests. (C) MRI images were acquired with a 7 T BioSpec spectrometer (Bruker, Germany). Tumor volumes were calculated for comparison between MBT2.cMYCshRNA.GFP and MBT2. ControlshRNA.GFP cells. 
regulated by shRNA, MBT-2 cells may be employed in the development of successful immunotherapies.

Transplantable syngeneic orthotopic bladder tumor models present two major drawbacks. These models exhibit a low rate of tumor uptake, and the disruption of the urothelium by electrocautery or chemical applications to facilitate tumor uptake produces particularly invasive and aggressive tumors. Weldon and Soloway [22] reported that tumor cell implantation occurred in $13 \%$ of untreated mice, while the administration of $N$-methyl- $N$ nitrosourea before tumor introduction resulted in a tumor uptake rate of approximately $60 \%$. Several modifications have been proposed to improve the success rate of orthotopic bladder tumor implantation. Shapiro et al. [23] reported a tumor uptake rate of approximately $60 \%$ with electrocauterization-induced mucosal injury before tumor introduction. In this modification, intravesical tumors were reduced by $24 \%$. Pretreatment with $\mathrm{HCl} / \mathrm{KOH}$ resulted in a tumor uptake rate of approximately 80 to $90 \%$, of which approximately $20 \%$ were extravesical tumors [24]. In an $\mathrm{HCl}$ pretreatment model, the entire bladder wall is exposed to acid, which leads to extensive mucosal injury. Chan et al. [25] reported a high mortality rate and extensive bladder wall inflammation with $\mathrm{HCl}$ pretreatment. Thus, this model is too aggressive to be used to study local treatment effects. MBT-2 tumors grow rapidly in the bladders of mice. The mean survival time of animals in the orthotopic bladder cancer model is approximately $20-40$ days. Thus, the rate of tumor development in the bladder and the animals' survival time must be controlled. In this study, we present a novel orthotopic bladder cancer model in $\mathrm{C} 3 \mathrm{H} /$ He mice in which the c-Myc expression of MBT-2 murine bladder cancer cells was inhibited to down-regulate the growth rate. However, $c-m y c$ is essential for p53-induced apoptosis in response to chemotherapeutic agent(s), so that chemotherapy acting through a mechanism inducing p53 expression is not efficacious in MBT2.cMYCshRNA.GFP cells stably down-regulating c-MYC proteins [26].

A number of reports address the role of the protooncogene $c-m y c$ in bladder tumors. Immunohistochemical staining with anti-c-MYC monoclonal antibodies revealed that the high expression of $c-m y c$ is associated with poorly differentiated bladder tumors [27]. With respect to copy number gains and amplifications in bladder cancer patients, alterations of the c-MYC gene, including copy number gains and amplifications, are linked to genetically unstable bladder cancers characterized by a high histologic grade and/or invasive growth [28, 29]. Therefore, this study inhibited the $c-m y c$ gene to reduce the rate cell proliferation and employed shRNA to downregulate $c-m y c$ expression. Furthermore, shRNA delivered by lentiviruses decreased the number of $c$-myc transcripts rather than completely eliminating them. Conclusively, the novel orthotopic bladder cancer model in $\mathrm{C} 3 \mathrm{H} / \mathrm{He}$ mice described here modified the $c$-myc expression level of MBT-2 murine bladder cancer cells to control their

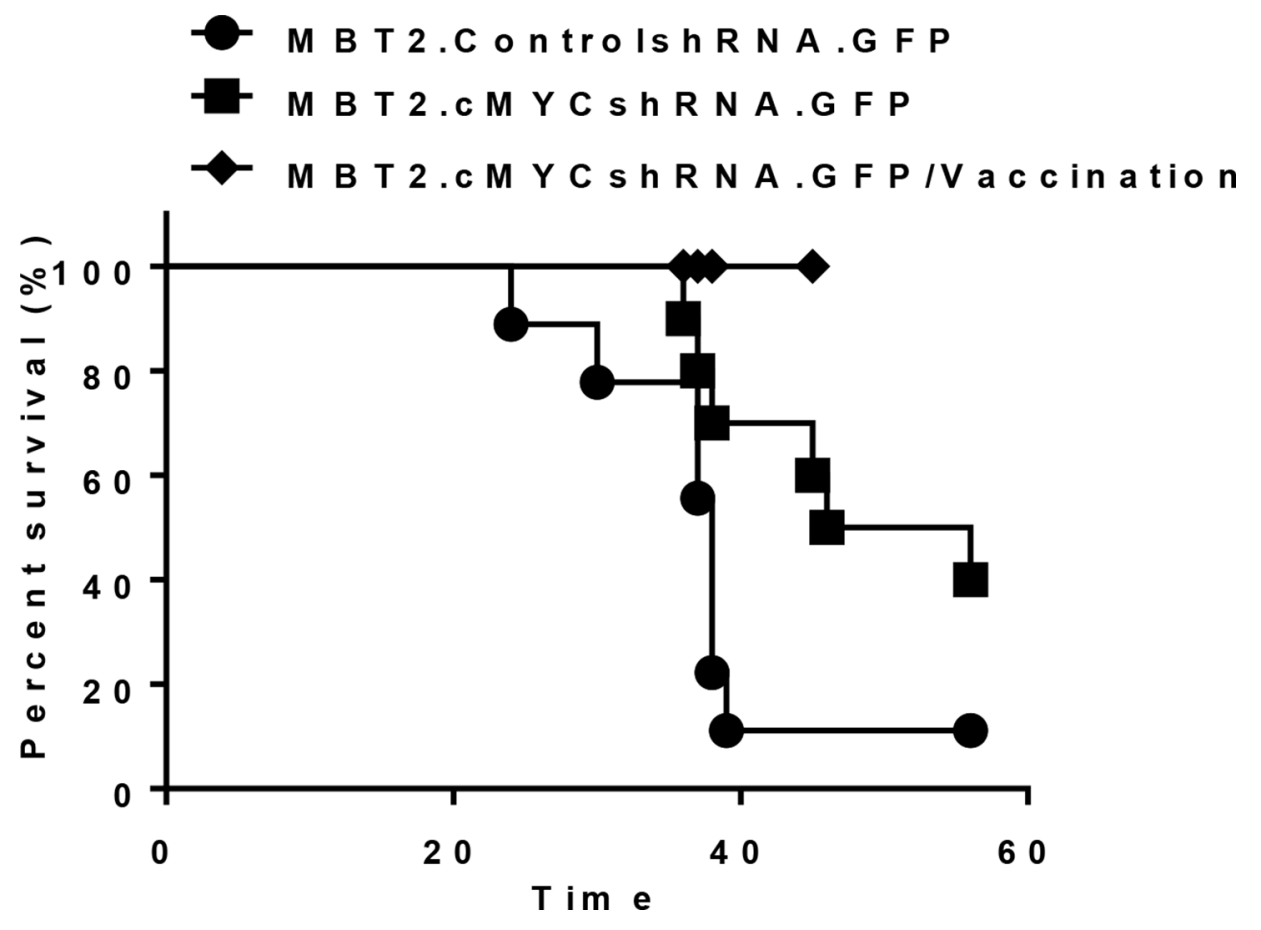

Figure 5: Prolonged survival of mice with orthotopic tumors in the bladder via the reduction of $c$-myc expression. Intravesical xenografts in the bladders of mice were established using MBT2.cMYCshRNA.GFP (-) or MBT2.ControlshRNA.GFP $(\bullet)$ cells. The long-term survival rate was determined $(P$ value, log-rank test). Furthermore, eight mice were included right after two subcutaneous vaccinations with $1 \times 10^{5}$ of MBT-2 cells that were pre-treated with 10 Gy irradiation ( $\bullet$ 
growth rate. This model is ideal for the evaluation of novel intravesical immunotherapies.

\section{MATERIALS AND METHODS}

\section{c-myc siRNA transfection}

MBT-2 cells from a mouse bladder cancer cell line were culture in RPMI 1640, 10\% FBS and 1\% penicillin/ streptomycin. Approximately $2 \times 10^{5} \mathrm{MBT}-2$ cells were prepared in a 6-well plate one day before transfection. Cells cultured to $60-80 \%$ confluence in a 6 -well plate were transfected with Lipofectamine 2000 (Invitrogen, Carlsbad, CA, USA) following the manufacturer's protocol. We used the following siRNA: 5'-AAA GAG CAA GAA GAU GAG GAA-3' and 5'-UUC CUC AUC UUC UUG CUC UUU TT-3' for $c$-myc (Sigma-Aldrich); 5'-CAG UCG CGU UUG CGA CUG GTT-3' and 5'-CCA GUC GCA AAC GCG ACU GTT-3' as scrambled siRNA (Sigma-Aldrich, St. Louis, MO, USA).

\section{Western blot analysis}

For western blot analysis, cells were lysed in RIPA buffer containing $10 \mathrm{mmol} / \mathrm{L} \mathrm{NaF}$ and $5 \mathrm{mmol} / \mathrm{L} \mathrm{VO} 4$ supplemented with protease inhibitors (Sigma-Aldrich). Approximately $30 \mu \mathrm{g}$ of protein from the soluble fraction was resolved on SDS-PAGE and transferred to a PVDF membrane. Anti-cMYC (Cell Signaling, CA) or anti-b-actin (Sigma-Aldrich) antibodies were added to the membranes and subsequently reacted with peroxidase-conjugated polyclonal antibody (Sigma-Aldrich). Antibodies were detected using an Enhanced Chemiluminescence Plus kit (GE Healthcare, Pittsburgh, PA, USA).

\section{Construction and transduction of lentiviruses}

We established stable MBT2.Luc cells by infecting lentiviruses harboring a luciferase gene under the SV40 promoter. A luciferase gene digested with NheI/HpaI pGL3 control vector (Promega, Wisconsin, WI, USA) was cloned into $X b a \mathrm{I} / H p a \mathrm{I}$ sites of the lentiviral vector pLoxLenti3.7 (Addgene, Cambridge, MA, USA), which produced pLL3.7/ Luc. Its expression was under the control of the SV40 promoter. Lentiviral particles produced from the pLL3.7/ Luc vector were generated by co-transfection with three plasmids, pMDLg/pRRE, pRSV-Rev and pCMV-G, using Lipofectamine 2000 (Invitrogen). MBT2.Luc cells were infected with lentiviruses expressing luciferase, and GFPpositive MBT2.Luc cells were sorted with a flow cytometer. To reduce $c$-myc expression, two oligonucleotides encoding c-myc-specific shRNA were designed and cloned into ApaI/ $X b a \mathrm{I}$ sites of the modified lentiviral vector pLoxLenti3.7, which produced pLL3.7/cMYCshRNA. The modified pLoxLenti3.7 vector exhibited the puromycin gene instead of GFP at pLoxLenti3.7. We selected the lentiviral vector that coded the $c-m y c$-specific shRNA by cutting and sequencing the strands with restriction enzymes. Next, lentiviral particles were generated with the same procedures employed for the production of the pLL3.7/Luc lentivirus. MBT-2 cells transduced with lentivirus pLL3.7/ cMYCshRNA were selected in the presence of $100 \mathrm{mg} /$ $\mathrm{mL}$ puromycin. Next, pLL3.7/cMYCshRNA.GFP was constructed. MBT2.cMYCshRNA cells were infected with lentiviruses from the pLoxLenti3.7 vector, which produced MBT2.cMYCshRNA cells that expressed GFP.

\section{Cell proliferation assay}

Approximately $3 \times 10^{3}$ cells were plated in 96-well plates, and their levels of proliferation were measured at the indicated time points using the Cell Counting Kit-8 (Dojindo Laboratories, Rockville, MD, USA). All experiments were performed in triplicate.

\section{Animal studies}

All animal experiments in this study were performed under specific pathogen-free conditions in accordance with the Guidelines for the Care and Use of Laboratory Animals of the National Cancer Center of the Republic of Korea. All animal experiments included five or six mice per group. To establish orthotopic bladder cancer models, eight-week-old female $\mathrm{C} 3 \mathrm{H} / \mathrm{He}$ mice were anesthetized with $1.75 \%$ isoflurane. A superficial 6-0 silk purse-string suture was placed around the urethral meatus before a lubricated $24 \mathrm{G}$ angio-catheter was passed through the urethra into the bladder. For the pre-treatment, $50 \mu \mathrm{L}$ of $0.1 \mu \mathrm{g} / \mathrm{ml}$ poly-L-lysine (Sigma-Aldrich) was instilled for 15 minutes, and the bladder was voided. A suspension of $2 \times 10^{6} \mathrm{MBT} 2$. Luc cells in $50 \mu \mathrm{L}$ of PBS was instilled into the bladder, and the purse-string suture was tied down for $2 \mathrm{~h}$. The presence of tumors in the bladder was confirmed by luminescence analysis. The same procedures were used in MBT2.cMYCshRNA cells.

\section{MRI experiments}

For the in vivo MRI studies, the animals were anesthetized with isoflurane. To visualize the tumor lesions, rapid MRI examinations were performed, which involved the T2-weighted rapid acquisition of 12 axial slices of the bladder with the relaxation enhancement (RARE) sequence. Acquisition parameters were as follows: repetition time $(\mathrm{TR})=2500 \mathrm{~ms}$, echo time $(\mathrm{TE})=35 \mathrm{~ms}, 256 * 256$ matrix, field of view $(\mathrm{FOV})=2 * 2 \mathrm{~cm}$, slice thickness $=0.7 \mathrm{~mm}$, and RARE factor $=8$. All MRI images were acquired with a $7 \mathrm{~T}$ BioSpec spectrometer (Bruker, Germany).

\section{ACKNOWLEDGMENTS AND FUNDING}

This study was supported by a grant from the National Cancer Center, Korea (NCC-1510170) and a 
grant from the Global Core Core Research Center (GCRC) grant (No. 2011-0030678) from NRF, MEST, Republic of Korea. We would like to thank Ms. Seo Hye-Hyun for her assistance in the development of the orthotopic bladder cancer model.

\section{CONFLICTS OF INTEREST}

None.

\section{REFERENCES}

1. Morales A, Eidinger D, Bruce AW. Intracavitary Bacillus Calmette-Guerin in the treatment of superficial bladder tumors. J Urol. 1976; 116:180-183.

2. Bracarda S, Altavilla A, Hamzaj A, Sisani M, Marrocolo F, Del Buono S, Danielli R. Immunologic checkpoints blockade in renal cell, prostate, and urothelial malignancies. Semin Oncol. 2015; 42:495-505.

3. Zhang ZT, Pak J, Shapiro E, Sun TT, Wu XR. Urotheliumspecific expression of an oncogene in transgenic mice induced the formation of carcinoma in situ and invasive transitional cell carcinoma. Cancer research. 1999; 59:3512-3517.

4. Gabriel U, Bolenz C, Michel MS. Experimental models for therapeutic studies of transitional cell carcinoma. Anticancer research. 2007; 27:3163-3171.

5. Horiguchi Y, Larchian WA, Kaplinsky R, Fair WR, Heston WD. Intravesical liposome-mediated interleukin-2 gene therapy in orthotopic murine bladder cancer model. Gene therapy. 2000; 7:844-851.

6. Kress TR, Sabo A, Amati B. MYC: connecting selective transcriptional control to global RNA production. Nat Rev Cancer. 2015; 15:593-607.

7. Amati B. Integrating Myc and TGF-beta signalling in cellcycle control. Nat Cell Biol. 2001; 3:E112-113.

8. Cole AM, Myant K, Reed KR, Ridgway RA, Athineos D, Van den Brink GR, Muncan V, Clevers H, Clarke AR, Sicinski P, Sansom OJ. Cyclin D2-cyclin-dependent kinase $4 / 6$ is required for efficient proliferation and tumorigenesis following Apc loss. Cancer research. 2010; 70:8149-8158.

9. Mickey DD, Mickey GH, Murphy WM, Niell HB, Soloway MS. In vitro characterization of four N-[4-(5-nitro2-furyl)-2-thiazolyl] formamide (FANFT) induced mouse bladder tumors. J Urol. 1982; 127:1233-1237.

10. Guerin M, Barrois M, Terrier MJ, Spielmann M, Riou G. Overexpression of either c-myc or c-erbB-2/neu protooncogenes in human breast carcinomas: correlation with poor prognosis. Oncogene Res. 1988; 3:21-31.

11. Mariani-Costantini R, Escot C, Theillet C, Gentile A, Merlo G, Lidereau R, Callahan R. In situ c-myc expression and genomic status of the c-myc locus in infiltrating ductal carcinomas of the breast. Cancer research. 1988; 48:199-205.

12. Papamichalis G, Francia K, Karachaliou FE, Anastasiades OT, Spandidos DA. Expression of the c-myc oncoprotein in human metaplastic epithelial cells of fibrocystic disease. Anticancer research. 1988; 8:1217-1222.

13. Dang CV. MYC on the path to cancer. Cell. 149:22-35.

14. Jeong KC, Kim KT, Seo HH, Shin SP, Ahn KO, Ji MJ, Park WS, Kim IH, Lee SJ, Seo HK. Intravesical instillation of c-MYC inhibitor KSI-3716 suppresses orthotopic bladder tumor growth. J Urol. 2014; 191:510-518.

15. Zhang N, Li D, Shao J, Wang X. Animal models for bladder cancer: The model establishment and evaluation (Review). Oncology letters. 2015; 9:1515-1519.

16. Oyasu R. Epithelial tumours of the lower urinary tract in humans and rodents. Food Chem Toxicol. 1995; 33:747-755.

17. Hadaschik BA, Black PC, Sea JC, Metwalli AR, Fazli L, Dinney CP, Gleave ME, So AI. A validated mouse model for orthotopic bladder cancer using transurethral tumour inoculation and bioluminescence imaging. BJU Int. 2007; 100:1377-1384.

18. Jager W, Horiguchi Y, Shah J, Hayashi T, Awrey S, Gust KM, Hadaschik BA, Matsui Y, Anderson S, Bell RH, Ettinger S, So AI, Gleave ME, et al. Hiding in plain view: genetic profiling reveals decades old cross contamination of bladder cancer cell line KU7 with HeLa. J Urol. 2013; 190:1404-1409.

19. Jager W, Moskalev I, Janssen C, Hayashi T, Awrey S, Gust KM, So AI, Zhang K, Fazli L, Li E, Thuroff JW, Lange D, Black PC. Ultrasound-guided intramural inoculation of orthotopic bladder cancer xenografts: a novel high-precision approach. PLoS One. 2013; 8:e59536.

20. Redelman-Sidi G, Glickman MS, Bochner BH. The mechanism of action of BCG therapy for bladder cancer--a current perspective. Nat Rev Urol. 2014; 11:153-162.

21. Witjes JA. Management of BCG failures in superficial bladder cancer: a review. Eur Urol. 2006; 49:790-797.

22. Weldon TE, Soloway MS. Susceptibility of urothelium to neoplastic cellular implantation. Urology. 1975; 5:824-827.

23. Shapiro A, Kelley DR, Oakley DM, Catalona WJ, Ratliff TL. Technical factors affecting the reproducibility of intravesical mouse bladder tumor implantation during therapy with Bacillus Calmette-Guerin. Cancer research. 1984; 44:3051-3054.

24. Chin J, Kadhim S, Garcia B, Kim YS, Karlik S. Magnetic resonance imaging for detecting and treatment monitoring of orthotopic murine bladder tumor implants. J Urol. 1991; 145:1297-1301.

25. Chan E, Patel A, Heston W, Larchian W. Mouse orthotopic models for bladder cancer research. BJU Int. 2009; 104:1286-1291.

26. Phesse TJ, Myant KB, Cole AM, Ridgway RA, Pearson H, Muncan V, van den Brink GR, Vousden KH, Sears R, Vassilev LT, Clarke AR, Sansom OJ. Endogenous c-Myc is essential for p53-induced apoptosis in response to DNA damage in vivo. Cell death and differentiation. 2014; 21:956-966. 
27. Kotake T, Saiki S, Kinouchi T, Shiku H, Nakayama E. Detection of the c-myc gene product in urinary bladder cancer. Jpn J Cancer Res. 1990; 81:1198-1201.

28. Lu Y, Liu P, Wen W, Grubbs CJ, Townsend RR, Malone JP, Lubet RA, You M. Cross-species comparison of orthologous gene expression in human bladder cancer and carcinogeninduced rodent models. Am J Transl Res. 2010; 3:8-27.
29. Nord H, Segersten U, Sandgren J, Wester K, Busch C, Menzel U, Komorowski J, Dumanski JP, Malmstrom PU, Diaz de Stahl T. Focal amplifications are associated with high grade and recurrences in stage Ta bladder carcinoma. International journal of cancer. 2010; 126:1390-1402. 